\title{
Human sensitivity to reinforcement feedback functions
}

\author{
PhIL ReED \\ Swansea University, Swansea, Wales
}

\begin{abstract}
Two experiments investigated human sensitivity to the temporally extended aspects of reinforcement schedules. Experiment 1 investigated human sensitivity to the extended and local aspects of three reinforcement schedules: variable ratio (VR), variable interval (VI), and variable-interval-plus-linear-feedback (VI+) schedules. Experiment 2 investigated this sensitivity on two reinforcement schedules: VI and VI+ schedules. In both experiments, there was evidence of sensitivity to the temporally extended aspects of the schedule: There were differences between the response rate on the VI+ and yoked-VI schedules, but no statistical difference in rates of response between the VR and VI+ schedules. The VI+ versus VI difference was much more pronounced when a lower response force needed to depress a lever was used. These results suggest that human subjects do show some sensitivity to temporally extended aspects of schedules of reinforcement.
\end{abstract}

Exploring the ability to integrate information over extended periods of time is important to the study of learning and behavior. Researchers have suggested that this ability underlies optimal performance, and the extent to which behavior is controlled by events occurring over an extended period of time is central to theoretical debate about instrumental performance (Schachtman \& Reed, 1998). In particular, the extent of this ability addresses a distinction between "molar" and "molecular" theorists (cf. Baum, 1981; Vaughan \& Miller, 1984). "Molar theorists" hold that behavior is guided by the integration of information about responses made and reinforcers earned, across temporally extended periods. Organisms learn to match their response rate output to the reinforcer rate input and, thus, come to perform optimally (see, e.g., Baum, 1981). In contrast, "molecular theorists" posit that reinforcement acts only to strengthen the immediately preceding behavior local to the delivery of reinforcement, and information over temporally extended periods is not integrated (e.g., Peele, Casey, \& Silberberg, 1984). For example, if response rates immediately prior to reinforcement are low, then strengthening the tendency to emit low local rates would reduce overall response rates. According to this view, optimal performance observed over extended periods is only a by-product of these local processes and does not depend on the ability to integrate information across extended periods (e.g., Vaughan \& Miller, 1984).

Schedules of reinforcement have often been employed to assess sensitivity to the temporally extended (molar) relationship between response output and reinforcement input, often characterized as the reinforcer feedback function. Demonstrating sensitivity to this molar characteristic of a schedule is important, since it would suggest that integration over time is a critical factor in instrumental perfor- mance. However, although the concept of molar sensitivity has currency in the literature at a theoretical level, the evidence in its support based on schedule performance is not without controversy. Under many conditions in which schedule performance suggests sensitivity to temporally extended aspects of the environment, molecular process may also lead to the same result. For example, response rates are higher on variable ratio (VR) schedules than on variable interval (VI) schedules (Peele et al., 1984). This result is compatible with the suggestion that responding is guided by sensitivity to the temporally extended reinforcer feedback function; higher response rates lead to higher reinforcer rates on VR schedules, but not on VI schedules. However, VI schedules also reinforce longer interresponse times (IRTs) than do VR schedules. Molecular theorists, like Peele et al., suggest that the reinforcement of particular IRTs is the prime determinant of schedule performance, such that if long IRTs are reinforced, then increased emission of this long IRT would lead to reduced overall response rates. On VI schedules, the probability of reinforcement for a response increases as the time since the previous response elapses, making longer IRTs more likely to be followed by reinforcement on VI schedules. This means that the molecular or local aspects of the schedule could also account for the lower response rates on VI schedules. Vaughan and Miller (1984) suggest that such confounds between the molecular and molar aspects of the contingencies make the use of simple schedules problematic, and that on more complex schedules, there is very little support for a molar view (see also Reed \& Schachtman, 1989).

The variable-interval-plus-linear-feedback (VI+) schedule (McDowell \& Wixted, 1986) is an important schedule to study in the investigation of molar sensitivity. 
It allows an assay of the difference between the impact of temporally extended features of the environment and features local to the time of reinforcement. On a VI+ schedule, overall reinforcer rates depend on overall response rates; thus, it has the molar features of a VR schedule. In contrast, long IRTs are reinforced, and it has the molecular features of a VI schedule. If subjects are sensitive to temporally extended information about response and reinforcement rate, performance on the VI+ schedule will be similar to that on a VR schedule. If information is not integrated over time and reinforcement acts to strengthen the immediately preceding behaviors, rates on a VI+ schedule should be like a standard VI schedule and be lower than those on a VR schedule.

On a VI+ schedule, the interval required before reinforcement becomes available is given by the equation: $a=$ $(i / n) \cdot b$, where $a=$ the interval required before a response will produce reinforcement; $i=$ the time from the last reinforcement to the present moment; $n=$ the number of responses made during $i$, and $b=$ the equivalent VR value. A numeric example may serve to illustrate how this schedule works. Assume that the VI+ schedule was to have the molar characteristics of a VR-30 schedule, that the time from the last reinforcer was $60 \mathrm{sec}$, and that 60 responses had been made during that time. In this case, the interval to reinforcement would equal $(60 / 60) \cdot 30=30 \mathrm{sec}$. During this $30 \mathrm{sec}$, at 60 responses/min, 30 responses would be emitted before the interval would time out. Alternatively, if only 30 responses had been made during that 60 -sec period, then the interval would be: $(60 / 30) \cdot 30=60 \mathrm{sec}$. During this $60 \mathrm{sec}$, if responses were being emitted at 30 responses/ min, 30 responses would be emitted prior to the reinforcer.

McDowell and Wixted (1986) required human subjects to press a lever for money reinforcement, and they found that rates of response on a VR and a VI+ schedule were equivalent to one another. They concluded that these data supported the suggestion that the molar aspects of the schedule controlled performance. However, a number of studies have attempted to investigate VI + schedule performance in rats, but none have noted the original effect obtained in humans (see Cole, 1999; Reed, Soh, Hildebrandt, DeJongh, \& Shek, 2000).

It is possible that the above discrepancies simply reflect a species difference. However, there are several problems with the original demonstration in humans that make it very important that this effect is replicated. As noted above, there is very little other unambiguous evidence for sensitivity to temporally extended aspects of the schedules (Schachtman \& Reed, 1998; Vaughan \& Miller, 1984). Hence, there is little strong evidence for the ability to integrate information across time on such contingencies. Critically, McDowell and Wixted (1986) did not run their subjects with a standard VI schedule without a linear feedback loop. Although such comparisons exist crossexperimentally, the use of such comparisons is difficult, and it may have been that their subjects (humans) would have responded at the same rate on any schedule to which they were exposed. Human subjects are known to exhibit great individual differences on how they respond on schedules of reinforcement, connected to the rules that they form about those schedules (see Lowe, 1979). Given the consistent failure to note the effect in nonhumans and the methodological problems noted in the original McDowell and Wixted study, an aim of the present study was to replicate the effect of molar sensitivity in humans and provide direct evidence of an ability to integrate information across time when exposed to schedules of reinforcement.

\section{EXPERIMENT 1}

The present study aimed to systematically replicate the experiment conducted by McDowell and Wixted (1986) including the appropriate control conditions - to ascertain whether sensitivity to the molar aspects of the schedules could be obtained.

\section{Method}

Subjects. Sixty-four participants served ( 27 male, 37 female, age range 18-27 years), all naive to the experimental procedure.

Apparatus. The apparatus consisted of a white board $(90 \times$ $60 \mathrm{~cm}$ ) with two $10-\mathrm{cm}$ response levers positioned equally distant $(10 \mathrm{~cm})$ from a central, recessed $10 \times 10 \mathrm{~cm}$ reinforcement hopper that was covered by a clear Perspex hinged flap, and that could be illuminated from behind by a jeweled houselight. Only the left lever was operative during the experiment. Weights were placed on the lever such that a force of $120 \mathrm{~N}$ would be required to depress the lever. Reinforcement consisted of one standard Smartie chocolate sweet, and this was delivered to the centrally located food hopper. The food hopper was illuminated whenever reinforcement was delivered.

Procedure. Participants received three 4-min trials of lever-press and magazine training on a VR schedule. The VR value was gradually increased on each trial from VR-5 to VR-15 to VR-30. Participants were then assigned randomly to four groups $(n=16)$; each group had similar mean response rates over the last two training trials (range 90-118 responses/min, $F<1$ ).

Group VR was exposed to a VR-30 schedule. Group VI+ was exposed to a VI+ schedule, such that the feedback function relating the response rate to the reinforcer rate was the same as a VR-30 schedule (as described above). The other two groups were exposed to a VI schedule; Group VI[yVR] was yoked to the VR schedule in terms of reinforcement rate, and Group VI[yVI+] was yoked to the $\mathrm{VI}+$ schedule in terms of reinforcement rate. One subject in Group VI[yVR] was yoked to a master subject in the VR group, and this pair was yoked throughout the experiment. The same occurred for the master and yoked subjects in Group VI+ and Group VI[yVI+]. The experiment was conducted across six 7-min trials, with an approximately 10-min break between the trials. Reinforcement rate was calculated for each master subject across each successive trial, and the yoked subject received the same reinforcement rate for each successive trial.

The following written instructions were attached to the equipment in front of the subjects and could be referred to throughout the experiment:

Please read these instructions aloud. This is a situation in which you can earn Smarties. At the end of the experiment, after all the results have been collected, the best performance will be rewarded with a sum of $£ 50$. You will be paid $£ 6$ anyway for doing the experiment. The experimenter will inform you when you can start earning smarties and when you cannot. You may eat the smarties as and when you please but you must always take every smartie that you do not eat out of the tray and place it in the container next to you. Please ignore the right lever. Please do not ask for additional information about what you are to do. Let the experimenter know when you are ready to begin. 


\section{Results and Discussion}

Figure 1 displays the group-mean response rates calculated over the final two trials of the experiment. These data were analyzed because they represented the terminal performance under the contingencies, and the data across these last two trials appeared to be stable (differences were less than $10 \%$ between the trials). There was a higher response rate for Group VR than for its yoked VI group (Group VI[yVR]). There was a higher response rate for the VR than for the VI+ group, and there was a higher response rate for Group VI+ than for Group VI[yVI+]. These data were analyzed by an ANOVA, with schedule as a between-subjects factor. A rejection criterion of $p<$ .05 was adopted for this and all subsequent analyses. This analysis revealed a significant main effect of schedule $[F(3,60)=4.84]$. Planned comparisons revealed statistically significant differences between the VR and its yoked VI group $[t(60)=3.00]$ and between the VI+ and its yoked VI group $[t(60)=1.84]$, but only a marginal difference between the VR and the VI+ groups $[t(60)=$ $1.73, .09>p>.08]$.

The reinforcement rates revealed that the yoking procedure was successful. Across the final two trials the group-mean reinforcements per minute (and $S D$ s) were as follows: Group VR $=4.54( \pm 1.77)$; Group VI[yVR] $=$ $4.46( \pm 0.99)$; Group VI+ $=1.65( \pm 0.71)$; Group $\mathrm{VI}[\mathrm{yVI}+]=1.33( \pm 0.46)$; both $p \mathrm{~s}>0.10$. An ANOVA revealed a significant main effect of schedule $[F(3,60)=$ 40.32]. Planned comparisons revealed no statistically significant differences between the VR and its yoked VI group $(t<1)$, or between the VI+ and its yoked VI group $(p>.10)$.

The group-mean reinforced IRT data (and $S D$ s) for the last two trials showed that Group VR had the shortest mean reinforced IRTs $(0.64 \pm 0.40)$, with the other three groups showing longer mean reinforced IRTs: Group VI $+=7.89$ ( \pm 6.24$)$; Group VI[yVI+] $=8.73( \pm 10.12)$; and Group $\mathrm{VI}[\mathrm{yVR}]=7.79( \pm 7.60)$. An ANOVA conducted on these data revealed a significant difference between the values $[F(3,60)=6.11]$. Subsequent Tukey's honestly significant difference (HSD) tests revealed pairwise differences between the VR and each of the other three groups, but no other pairwise difference was significant.

These data revealed the typical VR versus VI difference in response rate (see, e.g., Peele et al., 1984), indicating that humans were sensitive to the operation of different schedules. However, there was no statistically significant difference between the response rates generated by the VR and VI+ schedules, although both of these schedules generated higher response rates than did VI schedules yoked to them in terms of reinforcer rate. This lack of statistical difference between the VR and VI+ response rates was obtained despite the existence of different reinforcer rates between the groups on these schedules, as well as reinforcement of shorter IRTs on the VR in comparison with the VI+ schedule. The difference in response rates between the VR and VI+ schedules could be attributed to the difference in the reinforcer rate generated on these two schedules; the VR schedule had a higher rate than the $\mathrm{VI}+$ schedule.

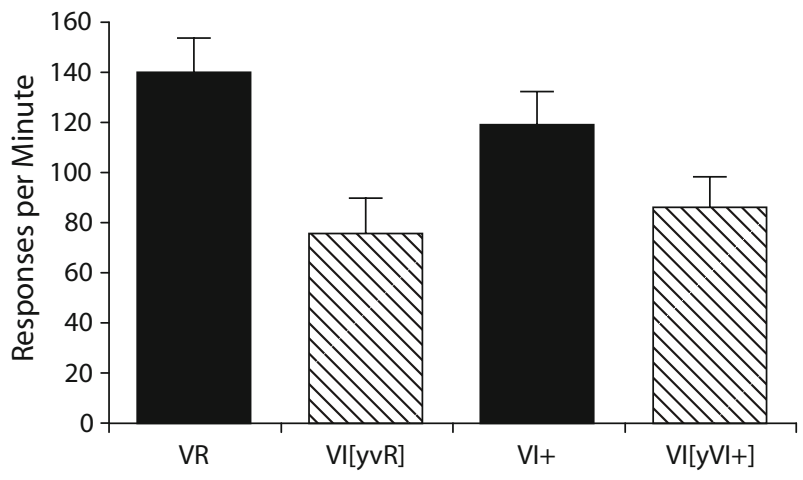

Figure 1. Results from Experiment 1. Group mean response rates (and standard errors) over the final two trials of the experiment. $\mathrm{VR}=$ variable ratio 30 schedule; $\mathrm{VI}+=$ variable interval with an equivalent feedback function to a VR30 schedule; $\mathrm{VI}[\mathrm{yVR}]=$ variable interval schedule yoked in terms of reinforcement rate to the VR schedule; $\mathrm{VI}[\mathrm{yVI}+]=$ variable interval schedule yoked in terms of reinforcement rate to the VI+ schedule.

These data offer support for the findings reported by McDowell and Wixted (1986) in their study of humans' sensitivity to molar aspects of the contingency. This finding was obtained after adopting the appropriate control conditions for establishing sensitivity to the VI+ schedule.

\section{EXPERIMENT 2}

Two findings of note emerged from the first experiment. A typical VR versus VI difference emerged in human subjects along with an indication that subjects were sensitive to the molar aspects of the schedules as indexed by a lack of difference between the VR and VI+ schedules. The second experiment attempted to further evaluate the finding that humans can be sensitive to molar aspects of the schedule by replicating the VI+ versus yoked VI difference that was noted in Experiment 1. By using this comparison, rates of reinforcement can be controlled, and despite equal reinforcer rates and reinforcement of similar IRTs, the VI+ schedule should produce higher response rates than a yoked VI schedule.

McDowell and Wixted (1986) and the present Experiment 1 employed a high response force $(120 \mathrm{~N})$ in their procedures. It may be that such a high response force makes it difficult to continue responding at high rates for prolonged periods of time. As Cole (1999) noted, a pause from responding on a VI+ schedule could result in a potentially catastrophic reduction in local reinforcement rate, leading to extinction. This sudden drop in responding when exposed to a VI+ schedule is noted in some of the rat subjects that were examined by Reed, Hildebrandt, DeJongh, and Soh (2003). It may be that evidence for sensitivity to the molar aspects of the schedule would emerge more easily if the response force was made low so that responding can occur without hindrance at high rates over a prolonged period of time. Experiment 2 investigated this possibility by manipulating response force; a 
high force and a low force condition were employed in this study with VI+ and yoked VI schedules. The latter two conditions were chosen since we assumed that if schedule differences were to be noted in this paradigm, they would most likely be observed between these schedules, and not between VR and VI+ schedules.

\section{Method}

Subjects and Apparatus. Sixty-four human subjects served in the present experiment (19 male, 45 female, age range 18-26 years). The apparatus was the same as that described in Experiment 1.

Procedure. Subjects were pretrained as was described in Experiment 1 before they were transferred onto the testing phase. The subjects were randomly assigned to four equally sized groups. Two groups responded on a VI+ schedule, with the feedback function of a VR-30 schedule. The other two groups responded on yoked VI schedules. One of the VI+ groups and one of the VI groups had a high response force, and the other had a low response force. Response force was determined by placing weights on the lever so that a high response force was $120 \mathrm{~N}$, and the low response force was $10 \mathrm{~N}$. The experiment was run for six 7-min trials as was described in Experiment 1.

\section{Results and Discussion}

Group-mean response rates over the final two trials can be seen in Figure 2. These trials were chosen for analysis because they represented terminal performance, and the data across these two trials showed relative stability (less than $10 \%$ variation). Inspection of these data reveals a higher response rate on the VI+ schedules than on the yoked VI schedules. This effect was more pronounced in the low than the high force conditions. A two-factor ANOVA (schedule vs. force) revealed statistically significant main effects of schedule $[F(1,60)=26.88]$ and force $[F(1,60)=7.62]$, along with a statistically significant interaction between the two factors $[F(1,60)=5.46]$.

The mean reinforcer rates per minute (and $S D \mathrm{~s}$ ) across the last two trials were: Group VI High $=2.11( \pm 0.93)$; Group VI+ High $=2.30( \pm 1.60)$; Group VI Low $=3.60$ $( \pm 2.36)$; Group VI+ Low $=3.74( \pm 2.21)$. A two-factor ANOVA (schedule vs. force) revealed a statistically significant main effect of force $[F(1,60)=11.25]$, but there was neither a significant main effect of schedule nor a significant interaction $\left[F_{\mathrm{s}}<1\right]$.

The group-mean reinforced IRTs (and $S D \mathrm{~s}$ ) on the last two trials were Group VI High $=3.21( \pm 2.59)$; Group VI+ High $=5.12( \pm 8.36)$; Group VI Low $=6.29( \pm 7.31)$; Group VI+ Low $=2.82( \pm 2.57)$. A two-factor ANOVA (schedule vs. force) revealed no statistically significant main effects or a significant interaction $(p s>.10)$.

Response rates were higher for the VI+ schedule than for the yoked VI schedules. The pattern of data from the high force conditions was similar to that found in Experiment 1 and appears to corroborate that found by McDowell and Wixted (1986). The same pattern of data was noted for the low force conditions, but was more pronounced. These data support the notion that the molar aspects of the schedule are important, since there were no statistically significant differences between the schedules in either reinforcement rate or reinforced IRTs across the conditions.

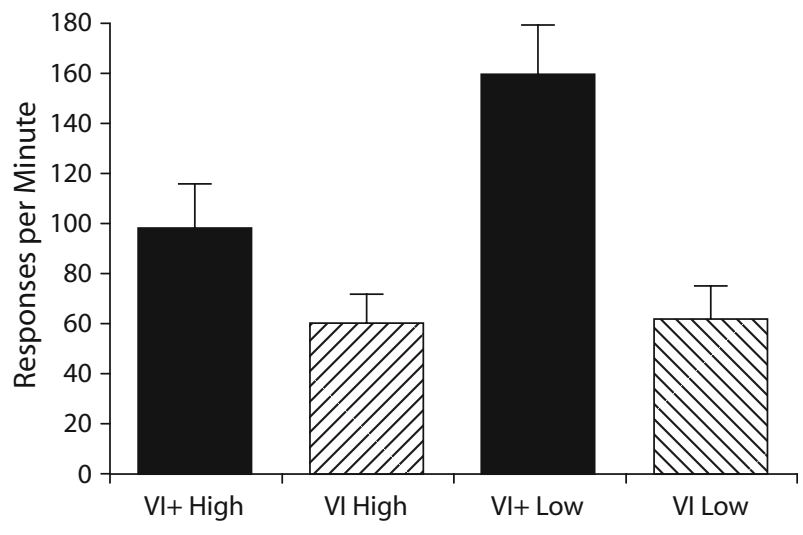

Figure 2. Results from Experiment 2. Group mean response rates (and standard errors) over the final two trials of the experiment. VI+ = variable interval with an equivalent feedback function to a VR30 schedule; VI = variable interval schedule yoked in terms of reinforcement rate to the VI+ schedule; Low $=$ low force condition $(10 \mathrm{~N})$; High $=$ high force condition $(120 \mathrm{~N})$.

\section{GENERAL DISCUSSION}

The present results support the findings reported by McDowell and Wixted (1986; see also Reed, 1999, 2001): Humans are sensitive to the molar aspects of the VI+ schedule. There was no statistical difference between the $\mathrm{VI}+$ schedule and the VR schedule in Experiment 1, despite differences in reinforcer rate and reinforced IRTs between the two schedules. Similarly, there was a difference between the response rate between a VI+ and a VI schedule in both Experiment 1 and Experiment 2, despite similar rates of reinforcement and similar reinforced IRTs on these schedules.

These findings of sensitivity to the molar aspects of the schedule in humans contrast with those reported by Cole (1999), Reed et al. (2000), and Reed et al. (2003) for rats. Of course, it is always difficult to compare across experiments-especially across studies using different species-however, without undue speculation, one aspect of the studies that differs between human and rat subjects concerns the rates of response emitted by the subjects. In all of the studies with human participants (i.e., McDowell \& Wixted, 1986; Reed, 1999, 2001; present Experiments 1 and 2), the response rate has been considerably higher than that noted in studies using nonhuman subjects (i.e., Cole, 1999; Reed et al., 2003; Reed et al., 2000). Thus, sensitivity to the VI+ schedule may only emerge when it is possible to maintain a high response rate. Although further empirical documentation is needed, there is some support for this view. The apparent greater difference between the VI+ and VI schedules in the low force condition of the present Experiment 2 is consistent with sensitivity to temporally extended aspects of schedules being associated with higher response rates. Cole (1999; see also Reed et al., 2000) suggested that the functioning of the VI+ schedule makes any pause in responding potentially catastrophic for local response rates. A reduction in response rate on the $\mathrm{VI}+$ schedule will generate an increased inter- 
reinforcement interval, which-if it becomes too longwill lead to extinction. If rates are not maintained across the schedule, then this could dramatically reduce response rates seen on the VI+ schedules. Such a catastrophic drop in responding was seen in some rats that were studied by Reed et al. (2003) when they were transferred to a VI+ schedule from a VR schedule.

The reason why human subjects display and maintain higher response rates than rat subjects is unclear. It could be that human subjects form verbal rules for themselves to allow this to occur (see Lowe, 1979). Human subjects could be capable of faster responding than rats, and key pecking by pigeons may provide data comparable to those of humans, since this response can be emitted at high rates. Alternatively, it could be that there is less scope for competing behavior in the human experimental situation in comparison with the nonhuman situation. It is certainly the case that the length of exposure to the schedules differs between the sets of experiments. In the present studies, the human participants received only six 7-min trials, whereas nonhuman subjects often receive months of 30- or 60-min sessions. Finally, there is possibly a real species difference that is responsible for the effect.

Whatever the reasons for the differences between the results of studies employing humans and rats, it is clear that the findings of McDowell and Wixted (1986) can be replicated and extended. Human sensitivity to the VI+ schedule has been demonstrated in the present series of studies using appropriate control conditions and procedures to generate sensitivity to schedules in humans. The present finding of molar sensitivity is important, since it is one of the few in literature, and such demonstrations have a bearing on the development of theory regarding the ability of organisms to integrate information across time.

\section{AUTHOR NOTE}

Some of these data were first presented at the Experimental Analysis of Behavior Group Meeting in London, April, 2001. Thanks to Mariane Soh and Gavin Nerveheese for collecting some of these data, and to Lisa A. Osborne for her support. Requests for reprints of this article should be addressed to P. Reed, Department of Psychology, University of Wales
Swansea, Singleton Park, Swansea, SA2 8PP, Wales (e-mail:p.reed@ swansea.ac.uk).

\section{REFERENCES}

BAUM, W. M. (1981). Optimization and the matching law as accounts of instrumental behavior. Journal of the Experimental Analysis of Behavior, 36, 387-403.

CoLE, M. R. (1999). Molar and molecular control in variable-interval and variable-ratio schedules. Journal of the Experimental Analysis of Behavior, 71, 319-328.

LowE, C. F. (1979). Determinants of human operant behaviour. In M. D. Zeiler \& P. Harzem (Eds.), Advances in analysis of behaviour: Vol. 1. Reinforcement and the organisation of behaviour (pp. 159-192). Chichester, U.K.: Wiley.

McDowell, J. J., \& WiXTED, J. T. (1986). Variable-ratio schedules as variable-interval schedules with linear feedback loops. Journal of the Experimental Analysis of Behavior, 46, 315-329.

Peele, D. B., Casey, J., \& Silberberg, A. (1984). Primacy of interresponse-time reinforcement in accounting for rate differences under variable-ratio and variable-interval schedules. Journal of Experimental Psychology: Animal Behavior Processes, 10, 149-167.

REED, P. (1999). Effect of perceived cost on judgments regarding the efficacy of investment. Journal of Economic Psychology, 20, 657-676.

REED, P. (2001). Schedules of reinforcement as determinants of human causality judgments and response rates. Journal of Experimental Psychology: Animal Behavior Processes, 27, 187-195.

ReED, P., HildebrandT, T., DeJongh, J., \& SoH, M. (2003). Rats performance on variable interval schedules with a linear feedback loop between response rate and reinforcement rate. Journal of the Experimental Analysis of Behavior, 79, 157-173.

REED, P., \& SCHACHTMAN, T. R. (1989). Instrumental responding by rats on free-operant contingencies with components that schedule response-dependent reinforcer omission: Implications for optimization theories. Animal Learning \& Behavior, 17, 328-338.

Reed, P., Soh, M., Hildebrandt, T., DeJongh, J., \& SheK, W. Y. (2000). Free-operant performance on variable interval schedules with a linear feedback loop: No evidence for molar sensitivities in rats. Journal of Experimental Psychology: Animal Behavior Processes, 26, 157-173.

Schachtman, T. R., \& ReEd, P. (1998). Optimization: Some factors that facilitate and hinder optimal performance in animals and humans. In W. T. O'Donohue (Ed.), Learning and behavior therapy (pp. 301333). Needham Heights, MA: Allyn \& Bacon.

Vaughan, W., \& Miller, H. L. (1984). Optimization versus response strength accounts of behavior. Journal of the Experimental Analysis of Behavior, 42, 337-348.

(Manuscript received May 10, 2006; revision accepted for publication August 31, 2006.) 